\title{
SIS mixer design for a broadband millimeter spectrometer suitable for rapid line surveys and redshift determinations
}

Frank Rice, Matthew Sumner, Jonas Zmuidzinas, R. Hu, Henry G. LeDuc, et al.

Frank Rice, Matthew Sumner, Jonas Zmuidzinas, R. Hu, Henry G. LeDuc, Andrew I. Harris, David Miller, "SIS mixer design for a broadband millimeter spectrometer suitable for rapid line surveys and redshift determinations," Proc. SPIE 4855, Millimeter and Submillimeter Detectors for Astronomy, (17 February 2003); doi: 10.1117/12.459710

Event: Astronomical Telescopes and Instrumentation, 2002, Waikoloa, Hawai'i, United States 


\title{
SIS mixer design for a broadband millimeter spectrometer suitable for rapid line surveys and redshift determinations
}

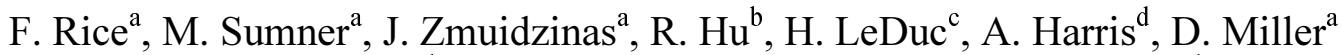 \\ ${ }^{\mathrm{a}}$ California Inst. of Technology; ${ }^{\mathrm{b}} \mathrm{U}$. of Michigan; ${ }^{\mathrm{c}} \mathrm{Jet}$ Propulsion Laboratory; ${ }^{\mathrm{d}} \mathrm{U}$. of Maryland
}

\begin{abstract}
We present some detail of the waveguide probe and SIS mixer chip designs for a low-noise 180-300 GHz doublesideband receiver with an instantaneous RF bandwidth of $24 \mathrm{GHz}$. The receiver's single SIS junction is excited by a broadband, fixed-tuned waveguide probe on a silicon substrate. The IF output is coupled to a 6-18 GHz MMIC lownoise preamplifier. Following further amplification, the output is processed by an array of $4 \mathrm{GHz}, 128$-channel analog autocorrelation spectrometers (WASP II). The single-sideband receiver noise temperature goal of 70 Kelvin will provide a prototype instrument capable of rapid line surveys and of relatively efficient carbon monoxide (CO) emission line searches of distant, dusty galaxies. The latter application's goal is to determine redshifts by measuring the frequencies of $\mathrm{CO}$ line emissions from the star-forming regions dominating the submillimeter brightness of these galaxies. Construction of the receiver has begun; lab testing should begin in the fall. Demonstration of the receiver on the Caltech Submillimeter Observatory (CSO) telescope should begin in spring 2003.
\end{abstract}

Keywords: SIS, submillimeter, mixer, spectrometer, broadband, heterodyne

\section{INTRODUCTION}

One of the most exciting recent discoveries in astronomy has been the detection of dozens of high redshift, very luminous galaxies using bolometer cameras on submillimeter-band telescopes. Dusty and obscured at short wavelengths, these galaxies have proven to be difficult to identify using optical and near infrared telescopes, rendering these instruments nearly useless for accurate redshift determinations ${ }^{1}$. The warm molecular gas in the star-forming regions of these galaxies exhibits prominent line emissions of the rotation spectrum of carbon monoxide (CO) at frequencies which are integral multiples of $115 \mathrm{GHz}$ and which extend well into the submillimeter range. The $180-300 \mathrm{GHz}$ atmospheric window provides an ideal frequency range for redshift determination using these emissions. Unfortunately, existing instruments have very narrow instantaneous bandwidths (no more than $4 \mathrm{GHz}$ ). Searching the entire $100 \mathrm{GHz}$ window would be prohibitively time consuming with such instruments. The motivation for this project has been to demonstrate a system which can overcome this limitation.

Superconductor-insulator-superconductor (SIS) devices are in principle capable of extremely large instantaneous bandwidths and noise temperatures within a few degrees of the quantum limit. We are developing a sensitive, broadband heterodyne spectrometer for the Caltech Submillimeter Observatory (CSO) which will be suitable for CO line searches and redshift determinations. The prototype receiver features a double-sideband design using a single SIS mixer excited by a full bandwidth, fixed-tuned waveguide probe. The 6-18 GHz IF output provides an instantaneous RF bandwidth of $24 \mathrm{GHz}$ (double-sideband). The IF signal will be analyzed by an array of analog autocorrelation spectrometers (WASP $\mathrm{II}^{2}$ ) providing a total of 384 channels. The wide instantaneous bandwidth and the single-sideband receiver noise temperature goal of 70 Kelvin will result in an instrument which should be capable of detecting CO line emissions with redshifts approaching $\mathrm{z}=2$. Additional applications of the instrument include rapid line surveys of galactic and extragalactic molecular clouds and on-the-fly mapping of tropospheric composition as part of a new NASA satellite mission concept under study at JPL to monitor atmospheric chemistry and pollution.

\section{SIS DEVICE AND DESIGN CONSIDERATIONS}

Heterodyne detection of the RF signal is accomplished using a SIS junction quasiparticle mixer. The SIS junction must have reasonably low leakage current and a sharp nonlinearity in its DC current-voltage characteristic in order to achieve high conversion gain and low noise. Because of its significant capacitance, an SIS junction's impedance rolls off at high 
frequencies. In a mixer design, the roll-off frequency of the SIS junction impedance must be approximately equal to or greater than the design RF bandwidth. Finally, the mixer must be able to accommodate the total RF noise power received without saturation.

Through analyses and trade studies, it was determined that a single-junction mixer design would be able to achieve the performance goals while using reasonably inexpensive and reliable SIS manufacturing technology available at JPL. The heart of the mixer is the single Nb-AIN-Nb SIS junction. The $1.3 \times 1.3$ micron junction's critical current density $\left(\mathrm{J}_{\mathrm{C}}\right)$ of about $16 \mathrm{kA} / \mathrm{cm}^{2}$ will provide a normal resistance $\left(R_{n}\right)$ of $8.5 \mathrm{ohms}$ and capacitance of 150 fF $\operatorname{arad}^{3}$. This value of $J$, was chosen so that the $R_{n} C$ product corresponds to an impedance roll-off frequency of $125 \mathrm{GHz}$, high enough that the bandwidth limitation imposed by the Bode-Fano theorem should be minimal. The large area and moderate current density (for AlN barriers) will require only inexpensive UV contact lithography and mature JPL technology, so junction quality will be high, and subgap-to-normal resistance ratios in excess of 20 should be attainable.

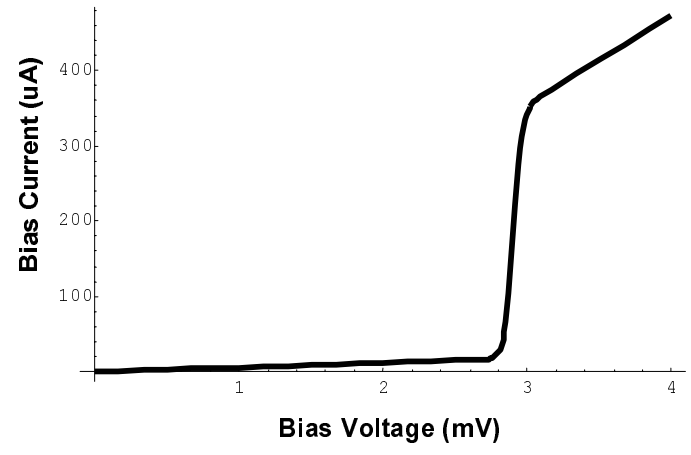

Figure 1: Modeled SIS DC IV characteristic curve for an $8.5 \mathrm{ohm} \mathrm{R}_{\mathrm{n}}$ device. This characteristic curve was used throughout the mixer design process to model the SIS junction. The curve was used to generate the fully nonlinear response of the heterodyne detector model using the SuperMix software package, including predictions of mixer noise and conversion gain. The SIS IV curve depicted is typical of Nb-AlN-Nb, moderate $J_{c}$ SIS junctions produced at JPL.

For the purposes of the receiver design, the assumed leakage current and nonlinearity of the SIS junction are slightly conservative and should be achievable with reasonable yields (Fig. 1). Although an $R_{n}$ of only 8.5 ohms may seem low compared to typical waveguide probe impedances of $30-50 \mathrm{ohms}$, it is well within the capability of a suitably designed $\mathrm{RF}$ matching network. This low value for $\mathrm{R}_{\mathrm{n}}$ simplifies the IF matching problem, increasing conversion gain, and mitigates saturation effects.

\section{LO: $200 \mathrm{GHz}$}

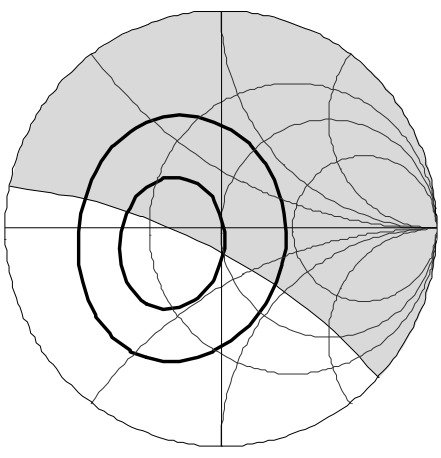

LO: $240 \mathrm{GHz}$

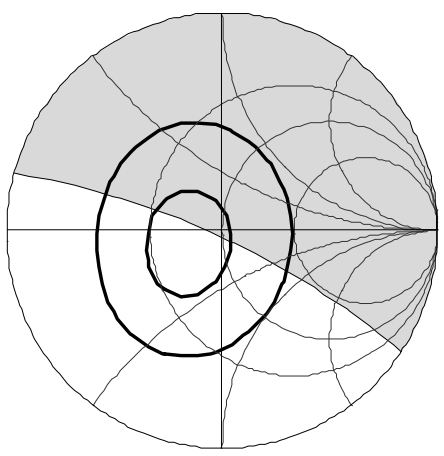

LO: $280 \mathrm{GHz}$

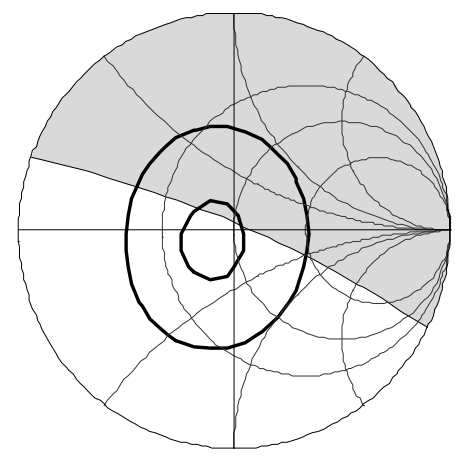

Figure 2: Effects of RF embedding impedance on mixer performance. The Smith charts show how mixer $\mathrm{T}_{\mathrm{n}}$ and stability vary with RF embedding impedance for a number of LO frequencies. The oval contours are for SSB mixer $T_{n}$ of $5 \mathrm{~K}$ and $10 \mathrm{~K}$ over the quantum limit; the gray regions denote the range of RF embedding impedance for which the mixer output impedance has a negative real part (IF instability). All charts are for upper sideband and an IF frequency of $10 \mathrm{GHz}$. The Smith charts are normalized to the SIS normal resistance, $\mathrm{R}_{\mathrm{n}}$.

Having chosen to use a single SIS device for the active mixing element, a study was conducted to determine the RF and IF embedding impedances seen by the SIS required for optimum noise performance and stability of the mixer. RF embedding impedance determines the noise temperature of the mixer as well as the IF stability. It must be carefully 
controlled or the SIS will present a negative real impedance to the IF circuit, resulting in receiver instability or even oscillation. Typical results of the RF embedding analysis (Fig. 2) demonstrate that the optimum RF embedding impedance should be less than $R_{n}$ and slightly capacitive; the impedance must be kept from going above $R_{n}$ or becoming inductive to avoid IF instability. As illustrated in Fig. 2, the best achievable mixer $T_{n}$ for an SIS with performance like Fig. 1 is about 3-5 Kelvin (single-sideband) over the quantum limit (note that Fig. 2 includes the SIS device capacitance as a part of the embedding impedance seen by the junction).

Once the RF embedding impedance is optimized for mixer $T_{n}$ and IF stability, the mixer conversion gain is controlled by the IF embedding impedance. Typical results of the IF embedding analysis (Fig. 3) show that excellent conversion efficiency is achievable, but RF reflection gain (instability) obtains when conversion gain is high. Excessive RF reflection gain can cause standing waves in the telescope optics, resulting in large gain variations for small changes in $\mathrm{RF}$ frequency. This problem can be mitigated by careful optical design; in principle, well-designed dewar and telescope optics should provide sufficient return loss so that a few $\mathrm{dB}$ of RF reflection gain from the SIS can be tolerated.
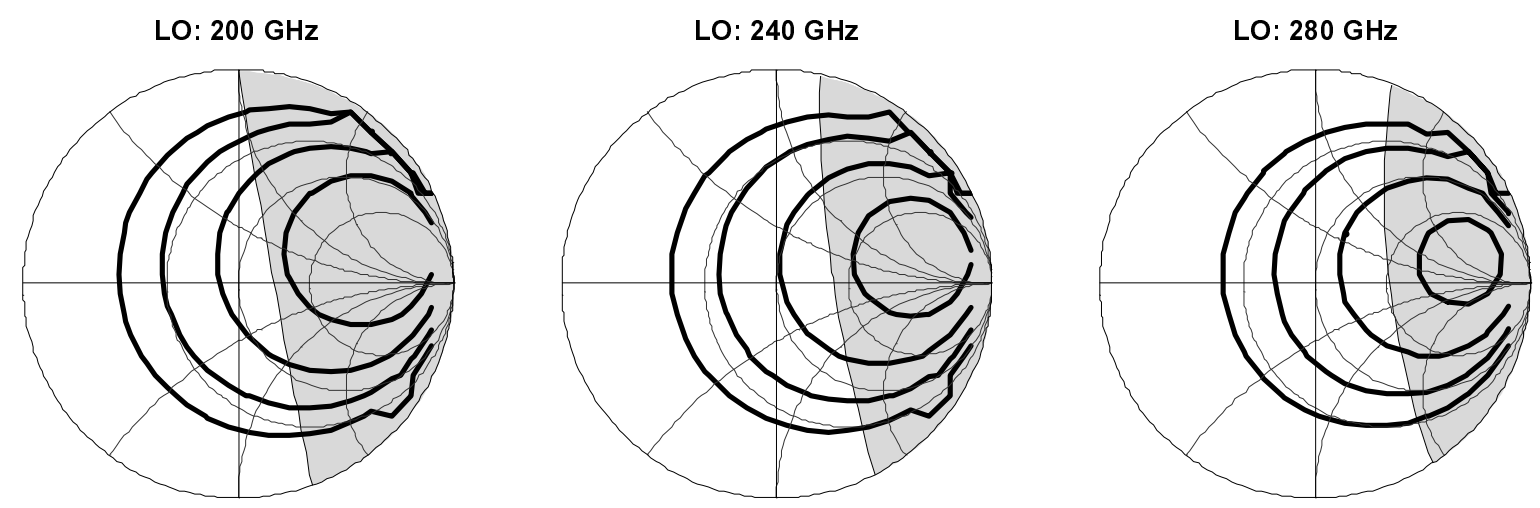

Figure 3: Effects of IF embedding impedance on mixer performance. The Smith charts show how mixer conversion gain $\left(\mathrm{S}_{21}\right)$ and RF stability vary with IF embedding impedance for a number of LO frequencies. The bold contours are for $\mathrm{S}_{21}=-2,0$, +2 , and $+4 \mathrm{~dB}$ (the smaller contours represent higher gains); the gray regions denote the range of IF embedding impedance for which the mixer input impedance has a negative real part (RF instability). All charts are for upper sideband and an IF frequency of $10 \mathrm{GHz}$. The assumed RF embedding impedance is that which minimizes mixer $\mathrm{T}_{\mathrm{n}}$ for the specified LO and IF frequencies. The Smith charts are normalized to twice the SIS normal resistance $\left(2 \times R_{n}\right)$.

\section{WAVEGUIDE PROBE}

Coupling from the receiver to the telescope is accomplished by a corrugated, cylindrical feed horn exciting a rectangular waveguide via a broadband cylindrical-rectangular transition section. A waveguide probe terminates the waveguide and couples RF radiation from it to the thin-film SIS detector circuit. The $37 \times 16$ mil (940×406 micron) waveguide has a cutoff frequency of $160 \mathrm{GHz}$. The $180-300 \mathrm{GHz}$ RF band (1.7:1 frequency ratio) includes $75 \%$ of the single-mode frequency range of the waveguide. Providing efficient waveguide to thin-film microstrip coupling over the large RF bandwidth is nontrivial, since the waveguide impedance varies by a 1.9:1 ratio and the waveguide wavelength $\left(\lambda_{\mathrm{g}}\right)$ varies by a 3.1:1 ratio over this bandwidth.

The performance of a waveguide probe can be characterized by its complex-valued reflection coefficient as seen by the RF circuitry $\left(\Gamma_{\text {probe }}\right)$. To achieve an efficient, broadband receiver design, $\Gamma_{\text {probe }}$ should:

- be slowly-varying and predictable over the RF band;

- be reasonably tolerant of machining errors and material property variations;

- correspond to a nearly real-valued impedance which can be matched to the SIS detector using a simple RF design;

- avoid the use of moveable waveguide elements which must be adjusted to tune the receiver during operation. 
Following a several-month study, design, and development effort a suspended-substrate waveguide probe design which satisfies all of the above requirements has been achieved. Derived from a design studied by Withington, et. al. ${ }^{4}$, the probe consists of a 90-degree radial sector on a dielectric substrate oriented so that the radial sector lies in a plane defined by the E-field of the $\mathrm{TE}_{10}$ mode and its propagation direction along the waveguide. A channel extending from the wall of the waveguide supports the substrate so that the probe is fixed in its proper position (Figs. 4 and 5). The design is notable in several respects:

- the substrate material is 25 micron thick silicon, which results in a significantly lower probe impedance than does a quartz substrate for this probe configuration;

- the probe has a radial stub geometry rather than the traditional rectangular, resulting in significant bandwidth improvement over earlier designs ${ }^{5}$;

- the substrate extends completely across the waveguide; RF energy is more efficiently coupled to the waveguide, reducing probe reactance;

- a small waveguide step upstream of the probe is used to further reduce probe impedance variation with frequency and to improve the tolerance of the probe performance to machining errors.
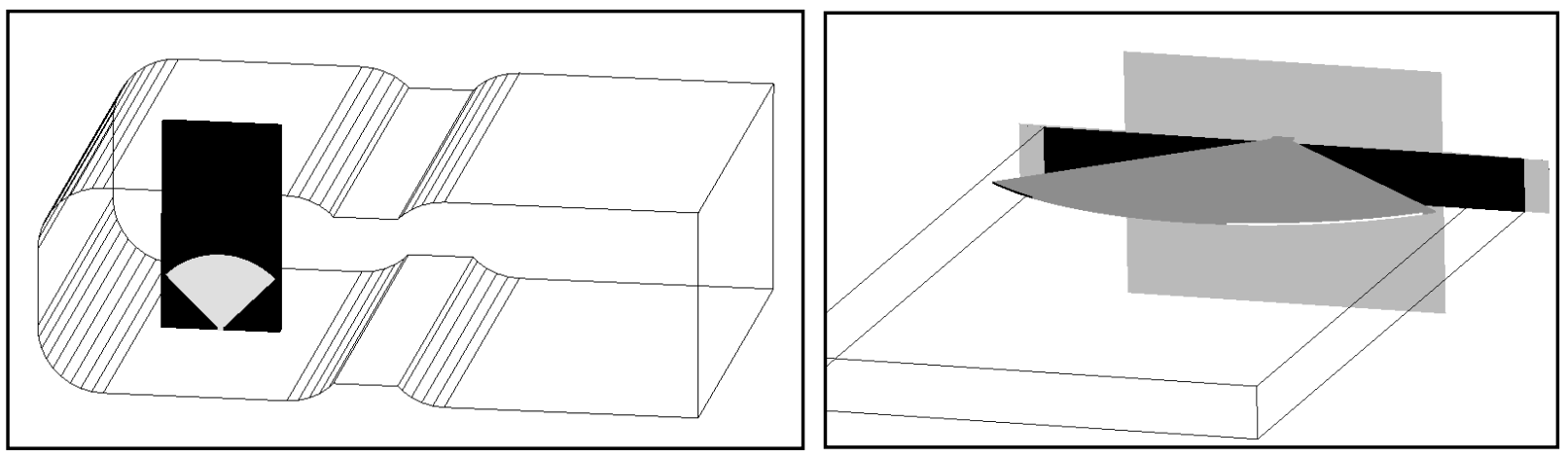

Figure 4 (left): General arrangement of the waveguide and probe. The $37 \times 16$ mil waveguide is terminated by a back short 11 mils behind the probe centerline. The silicon substrate (dark gray) is 230 microns wide and 25 microns thick $(9.1 \times 1.0$ mil) and extends completely across the height of the waveguide. The 90-degree radial probe (light gray) has a 150 micron (5.9 mil) radius and consists of a 0.40 micron thick niobium film deposited on the surface of the substrate; it is terminated in a thin-film microstrip transmission line connected to the RF detector circuitry. The short reduced-height section of waveguide just upstream of the probe serves as a capacitive tuning element and significantly improves the probe performance over the wide bandwidth required. All waveguide features are designed to be machined with a 5 mil fillet radius as shown.

Figure 5 (right): Detail showing how the probe substrate is mounted in a channel extending from the waveguide wall. The silicon substrate (dark gray and gray outline) is shown protruding from the waveguide wall. The light gray area shows the channel within which the substrate is suspended. The channel includes air gaps extending 1.0 mil above and 2.0 mil below the substrate, which ensure that the cutoff frequency for wave propagation within the channel remains above the operating RF range of the receiver. The substrate and its channel extend approximately $1 \mathrm{~mm}$ (40 mil) beyond the waveguide wall.

Probe development required several hundred hours of detailed electromagnetic (EM) field simulations followed by optimization of the waveguide back short and tuning element. The EM field simulations used Ansoft $\mathrm{HFSS}^{\circledR}$, a commercial program. The optimizations were accomplished using the SuperMix library, a Caltech-developed software package described later. Design of the substrate channel was guided by a theoretical analysis of EM wave propagation in partially-filled rectangular waveguide.

Final calculation of the predicted probe performance (Fig. 6) was accomplished using an HFSS analysis of the complete waveguide and probe assembly using the manufacturing drawings. These results have been validated by lab network analyzer measurements of a scale model probe of a very similar design. As can be seen in the figure, predicted probe impedance is very nearly constant and real over the entire $180-300 \mathrm{GHz}$ RF frequency range, with an average value of $36+\mathrm{j} 3$ ohms. 


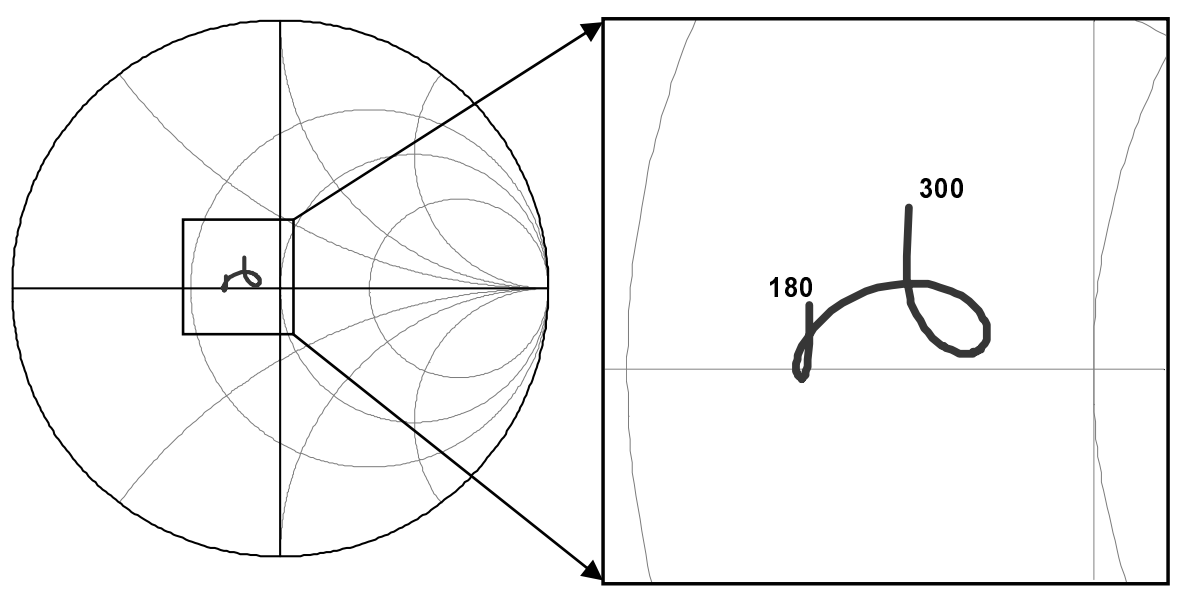

Figure 6: Waveguide probe performance. Predicted performance of the waveguide probe design shown in Fig. 4, as calculated using Ansoft HFSS and validated using a laboratory scale model of a similar design. The complex-valued reflection coefficient as seen by the RF circuitry, $\Gamma_{\text {probe}}$, is plotted on a Smith chart normalized to 50 ohms. The curve covers $180-300$ $\mathrm{GHz}$, the operating range of the receiver. $\Gamma_{\text {probe }}$ stays well within 0.1 of its average value (corresponding to an impedance of $36+\mathrm{j} 3 \mathrm{ohms}$ ) over that frequency range.

\section{RF MATCHING NETWORK}

The SIS junction is coupled to the waveguide probe using an RF matching circuit which must tune out the SIS device's large capacitance and transform the probe impedance so that the SIS sees the optimum RF embedding impedance. It must accomplish these tasks over the entire RF frequency range of the receiver, keeping the RF embedding impedance seen by the SIS under tight control in order to minimize mixer noise temperature while avoiding mixer instabilities and oscillation at the IF output. The RF circuit should be compact and simple in order to reduce its capacitive load on the IF output circuit and its loss at RF frequencies. Finally, the RF design must be tolerant of typical UV contact lithography alignment and etching errors with only minor performance degradation.

Straightforward analysis of the RF embedding impedance requirements demonstrates that a simple parallel or series inductance tuning element (to resonate out the SIS device's parallel capacitance) is inadequate to achieve the broadband, fixed-tuned performance required. Analyses and optimizations of various networks have demonstrated that a properlydesigned C-L-C "pi" network can meet the bandwidth goal. Additional impedance transformer stages are needed to lower the $36 \mathrm{ohm}$ probe impedance to the $\mathrm{R}_{\mathrm{n}} / 2$ or so required at the input to the pi network.

The final RF matching network (Fig.'s 7 and 8 ) is implemented in thin-film, superconducting, microstrip circuitry using niobium ground plane and wiring layers separated by a $\mathrm{SiO}$ dielectric layer. The circuitry is deposited on the waveguide probe substrate along with the probe itself and the SIS junction. Although conceptually the RF circuit is a 1/4-wave transmission line transformer followed by a lumped-element LC ladder (Fig. 7), the analysis and optimization were performed using a full model of the thin-film, superconducting, microstrip circuitry and SIS device implemented with the SuperMix software package. The effects of stray capacitance and inductance at the circuit junctions and corners were analyzed using the HFSS 3-D electromagnetic simulator and were included in the SuperMix model. The RF embedding impedance of the design (Fig. 9) is nearly optimum throughout the RF frequency range. The resulting mixer noise performance should remain within a few degrees of the quantum limit throughout the design RF and IF bandwidth (Fig. $10)$. 


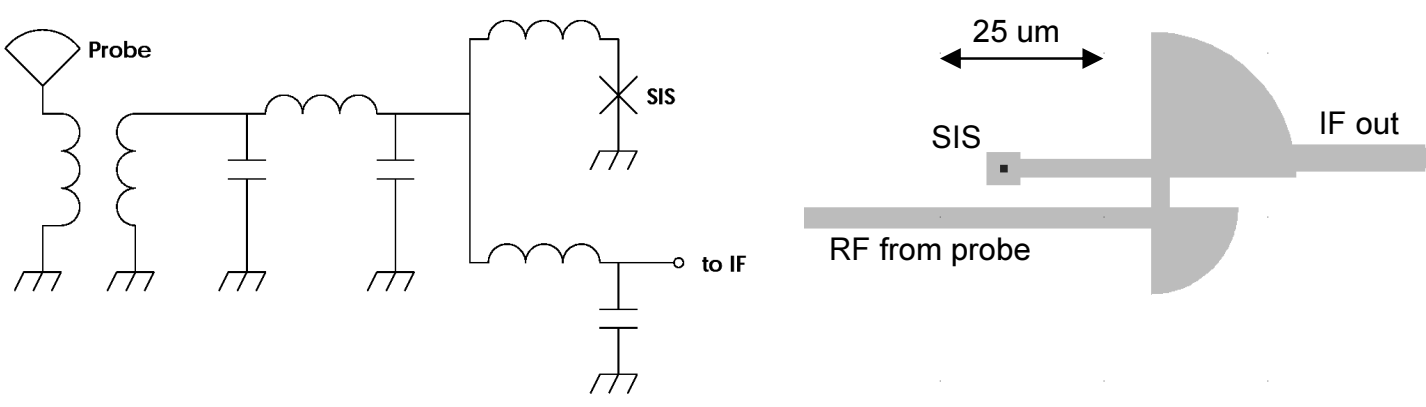

Figure 7 (left): Conceptual equivalent circuit of the RF matching network. The waveguide probe is connected to the SIS using a $1 / 4$-wave transformer followed by a lumped-element LC ladder. The capacitance associated with the SIS junction is not shown as a separate element in this figure.

Figure 8 (right): Thin-film microstrip layout of the RF matching network. The microstrip transmission line coming in from the left carries the LO and RF signals from the waveguide probe; only the final portion of its 108.6 micron length is shown. The radial stubs serve as parallel capacitors, the short sections of narrow microstrip as series inductors (Fig. 7). The IF output is extracted from the fan end of a radial stub, which is a particularly low impedance point in the RF circuit.
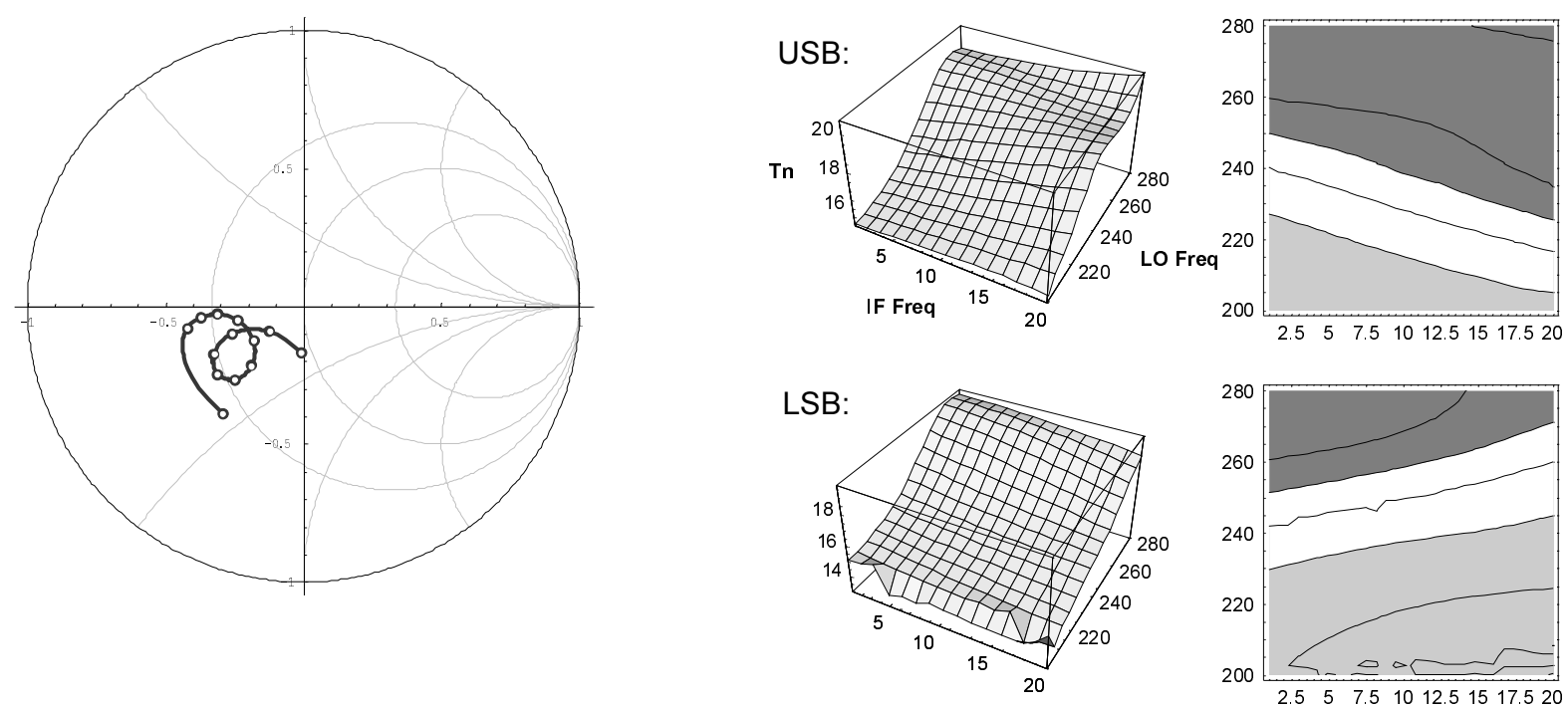

Figure 9 (left): Smith chart showing the design RF embedding impedance seen by the SIS, resulting from the circuit made up of the waveguide probe, RF matching network, and SIS capacitance. The frequency range is 180-300 GHz; the circles are spaced at $10 \mathrm{GHz}$ intervals. The Smith chart is normalized to the SIS $\mathrm{R}_{\mathrm{n}}=8.5 \mathrm{ohms}$. Compare with the optimal embedding impedance charts in Fig. 2.

Figure 10 (right): Predicted mixer noise performance. Shown are 3-D and contour plots of mixer single-sideband $T_{n} v$. LO and IF frequencies for both upper sideband (USB) and lower sideband (LSB) detection. Contours are at 1 Kelvin intervals; $T_{n}<16 \mathrm{~K}$ (including quantum noise!) are in light gray, $T_{n}>18 \mathrm{~K}$ are in dark gray. Mixer noise is generally less than $6 \mathrm{~K}$ over the quantum limit throughout the frequency range of the receiver.

\section{RF CHOKE AND IF MATCHING NETWORK}

The IF output signal from the SIS junction must be coupled efficiently to the load presented by the low noise IF preamplifier. At the same time, RF energy must be isolated from this low-frequency output, or receiver sensitivity will 
suffer. A single thin-film circuit fabricated along with the RF matching network on the surface of the waveguide probe substrate is used to perform both the IF matching and RF isolation services.
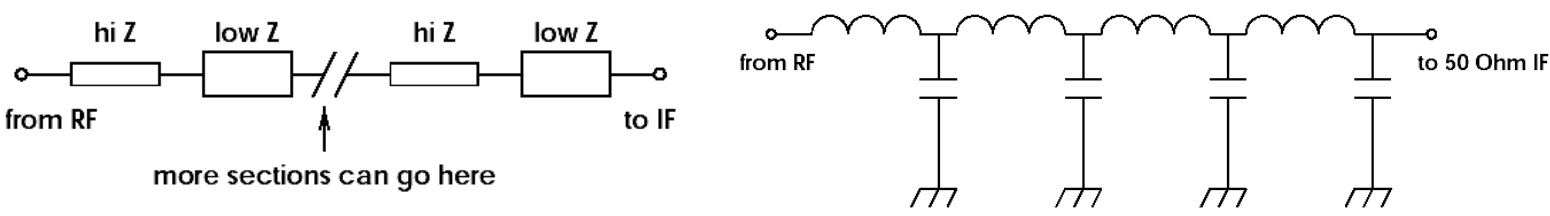

Figure 11: RF choke and IF matching network. By cascading high impedance-low impedance pairs of $1 / 4$-wave transmission lines at RF frequencies, effective isolation of the IF output from the RF circuit can be achieved (left). At the much lower IF frequencies, these transmission line sections behave as a lumped-element LC ladder network (right). The impedances of the transmission lines can be chosen to optimize the IF load matching. A total of four high-low pairs are used in the receiver circuit, with a 1/4-wave design frequency of $287 \mathrm{GHz}$ and a characteristic impedance of $90 \mathrm{ohms}$ for the high impedance sections.

The IF output is extracted from the fan end of a radial stub in the RF circuit (Fig. 8). The fan end of the radial stub is convenient because the impedance of the RF matching network is quite low at that point of the circuit. Consequently effective RF isolation can be achieved by ensuring that the IF circuit presents a high impedance relative to this very low impedance part of the circuit. Generating a high RF impedance at the input to the IF circuit is achieved by cascading a series of high and low impedance 1/4-wave transmission lines (Fig. 11). Each high/low pair transforms its load impedance by a factor equal to the square of the impedance ratio of the pair, so quite high impedances can be maintained at the input of this RF choke structure even though the IF load may present large impedance variations within the RF bandwidth.
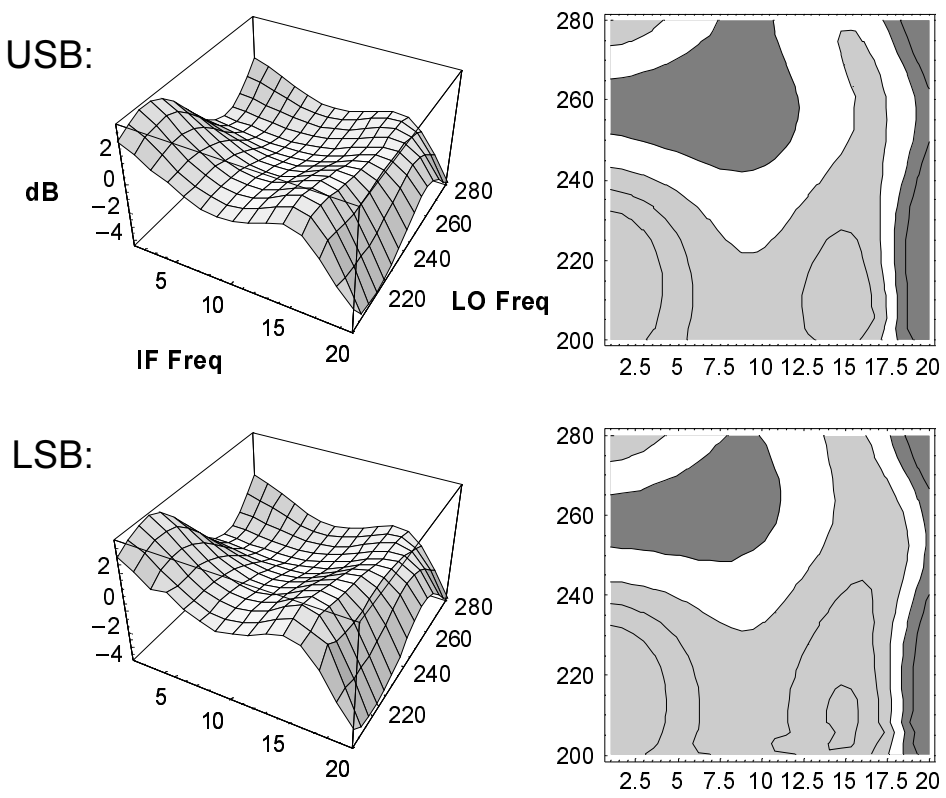

Figure 12: Predicted mixer conversion efficiency. Shown are 3-D and contour plots of mixer single-sideband conversion gain (dB) v. LO and IF frequencies for both upper sideband (USB) and lower sideband (LSB) detection. Contours are at $1 \mathrm{~dB}$ intervals; gains $>0 \mathrm{~dB}$ are in light gray, gains $<-1 \mathrm{~dB}$ are in dark gray. The mixer provides conversion gain over most of the operating frequency range of the receiver.

At IF frequencies the cascade of $1 / 4$-wave lines behaves as a lumped-element LC ladder structure, since IF wavelengths are an order of magnitude longer than RF wavelengths. As a result, the RF choke structure can also be used to match the 
IF load to the SIS device, tuning out the reactances of the SIS capacitance and the RF matching network in the IF band and maximizing mixer conversion gain. Design of the IF structure involves selecting the number of sections, choosing the $1 / 4$ wavelength RF design frequency, and selecting the characteristic impedance of each section in order to maximize the conversion gain of the mixer when driving a specified IF load while maintaining a sufficient RF stability margin and isolation of the IF circuit from the RF signal. Since high impedance lines are required, the design uses a combination of $\mathrm{CPW}$ and microstrip lines in the choke. Using SuperMix to perform the circuit optimization, the design has good predicted conversion efficiency over the entire receiver operating range, minimizing the impact of IF amplifier noise performance on the overall sensitivity of the receiver (Fig. 12).

\section{CHIP LAYOUT AND MIXER BLOCK}

The SIS and RF circuitry are deposited directly onto the silicon dielectric substrate supporting the waveguide probe using UV contact lithography. The thin-film superconducting circuitry is deposited on the silicon substrate in three layers: a 0.2 micron niobium ground plane, a 0.35 micron $\mathrm{SiO}$ dielectric layer, and a 0.4 micron top niobium wiring layer. The waveguide probe itself will be laid down as a part of the niobium wiring layer, whereas the ground plane layer will end at the waveguide wall. The SIS junction is connected to the top wiring using a 5 micron square pad to provide for lithography alignment errors. Two additional gold layers are deposited to complete the chip: a bond pad layer for the DC bias and IF output connections and a beam lead layer to connect the niobium ground plane to the waveguide block structure (Fig. 13).

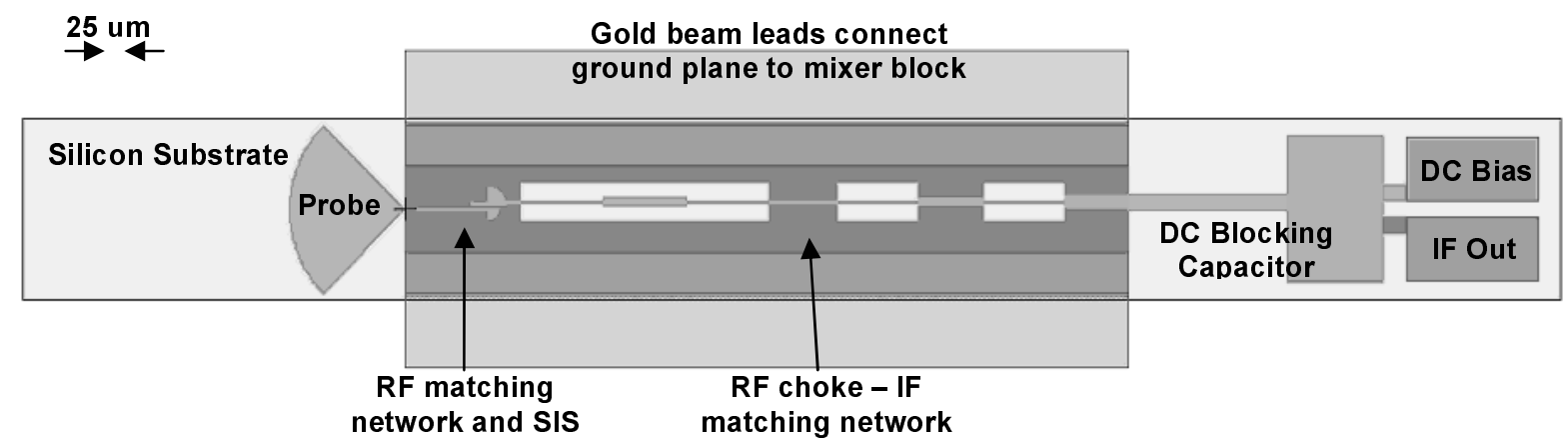

Figure 13: Probe-mixer chip layout. The probe substrate is 25 micron thick silicon, 230 microns wide $\times 1600$ microns long. The circuitry is applied to the top of the substrate using UV contact lithography. The five layers: niobium ground plane; SiO dielectric; niobium wiring; gold bond pads; gold beam leads. The waveguide probe is part of the wiring layer, connecting to the RF matching network. The RF choke uses CPW sections to increase line characteristic impedance; these sections can be identified by the rectangular gaps in the ground plane layer. Minimum wiring line thickness is 2.7 microns.

The ground plane layer must have the same electric potential as the waveguide wall surface at the mouth of the substrate channel or the probe performance will be seriously degraded. This is accomplished by extending the ground plane conductor laterally beyond the edges of the substrate material using beam lead technology similar to that developed at JPL in support of their high frequency multiplier development. The beam leads are electrically mated to the waveguide block structure to ensure that a good ground connection is achieved.

The mixer chip is mounted in a split-waveguide block. The block (Fig. 14) is attached to the corrugated feed horn and also contains a small circuit board for SIS DC bias components. The IF output from the block is via a high-frequency Ktype connector which mates directly to the input connector on the low noise IF preamplifier housing. The mixer chip sits in a channel machined in one half of the waveguide block. Its beam leads rest on the block surface on either side of the channel, and are pinched between the two block halves when mated together. The block requires only straightforward mechanical milling; fillet radii in the waveguide are all a generous 5 mils and the waveguide structure is designed so that the receiver performance is tolerant of expected machining accuracies. 

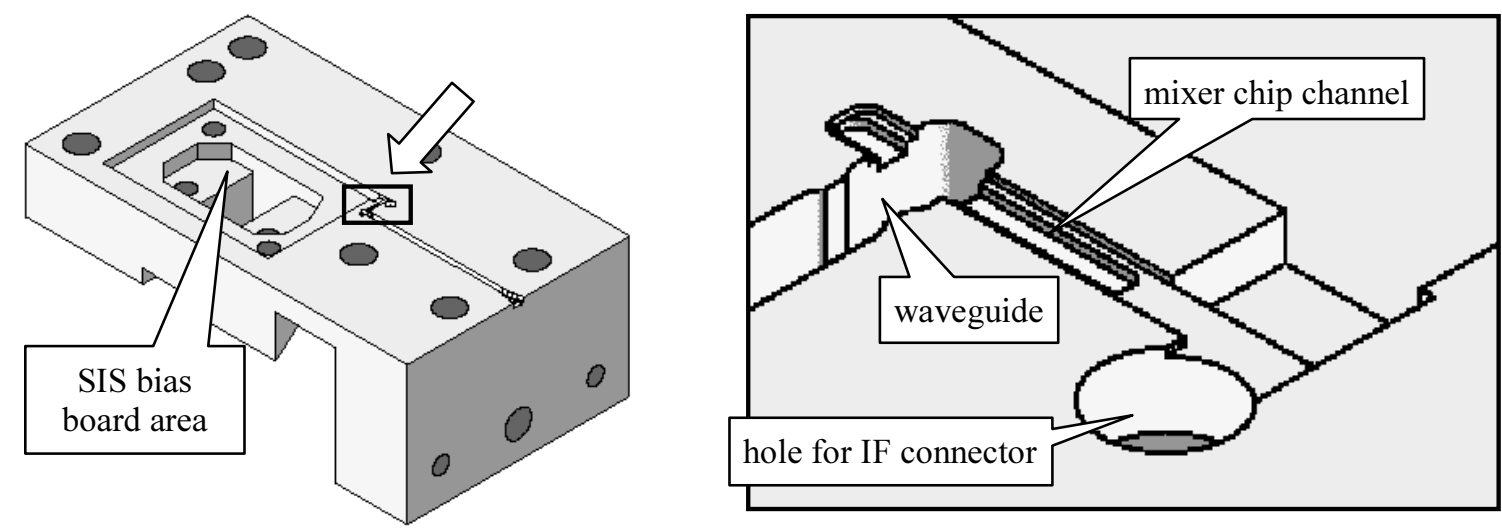

Figure 14: The waveguide block showing the mounting area for the mixer chip. The close-up view on the right uses a viewing angle different from that of the entire block half shown on the left; the arrow indicates the viewing perspective for the close up view. The IF output is via a glass bead to a K-type connector mounted to the bottom surface of the mixer block; the center conductor pin of the glass bead sticks up through the cylindrical hole adjacent to the chip channel. A wire bond connects the pin to the IF output pad on the mixer chip.

\section{SUPERMIX}

An important part of mixer design effort has included extensive enhancement of the SuperMix software package developed at Caltech to aid in the design and optimization of superconducting submillimeter wave receivers. Designed to run under UNIX or Linux, SuperMix is an extensive C++ software library containing over 47,000 lines of code ${ }^{6}$.

The SuperMix class library and its associated programs allow a researcher to write, compile, and run sophisticated circuit simulations of arbitrary complexity. SuperMix provides a complete set of circuit elements suitable for frequencydomain simulations from DC to the terahertz range. The library includes models of SIS quasiparticle tunnel junctions and physical transmission line components such as microstrip and CPW lines. The physical transmission line objects can be built up from layers of normal metal and superconducting films and real dielectrics. SuperMix can perform full harmonic balance calculations of SIS quasiparticle receiver designs of arbitrary complexity as well as mixer gain and noise analyses using any number of harmonics and including any number of SIS junctions ${ }^{7}$.

The SuperMix package includes a sophisticated multi-parameter optimizer. Users can tailor the optimizer's error function to their exact needs, and the optimizer can then control any set of device parameters in order to refine a circuit design. To achieve this level of flexibility, SuperMix contains a rather complete numerical math library for manipulation of complex-valued matrix, vector, and scalar functions and objects. It includes robust linear algebra, interpolation, integration, root finding, and minimization routines. More details regarding SuperMix may be found at the SuperMix website: http://www.submm.caltech.edu/supermix/.

\section{WIDEBAND CRYOGENIC MMIC LOW-NOISE IF PREAMPLIFIER}

The wideband mixer IF output must be amplified using a low noise preamplifier (LNA) with bandwidth and noise performance which will not compromise the overall performance of a receiver. The amplifier must be stable and have predictable and moderate input impedance over a bandwidth of $50 \mathrm{GHz}$ (to avoid out-of-band oscillation or output saturation of the SIS). It should have high gain and a flat frequency response over the 6-18 GHz IF bandwidth as well as noise temperatures below 10 Kelvin across the IF band.

Microwave monolithic integrated circuit (MMIC) technology employing indium phosphide (InP) high electron mobility transistor (HEMT) active elements has been identified as the most promising route to achieving the broadband, low noise performance goals of the LNA. The current device being developed is based on the JPL/Caltech WBA8T series MMIC design, fabricated at TRW. This chip has a 75 micron thick substrate measuring 750 by 2000 microns and has 4 
InP HEMT stages. In order to operate at $20 \mathrm{GHz}$ a 150 micron gate width HEMT is used, resulting in good gain over an excellent bandwidth of 2-20 GHz for the four transistor cascade. After characterizing the performance and limitations of current designs, the project has entered a period of design refinement through successive phases of modeling, building, testing, analyzing, and fixing in association with teams at JPL and TRW.

The current design, WBA8T-E, has a flat gain response over the 6-18 GHz IF band, with gain exceeding $20 \mathrm{~dB}$. The input return loss is about $10 \mathrm{~dB}$ across this same band ( $50 \mathrm{ohm}$ source). The amplifier noise temperature at $4 \mathrm{~K}$ is less than $13 \mathrm{~K}$ (50 ohm source) over the IF band, and less than $10 \mathrm{~K}$ from 8 to $15 \mathrm{GHz}$ (Fig. 15).
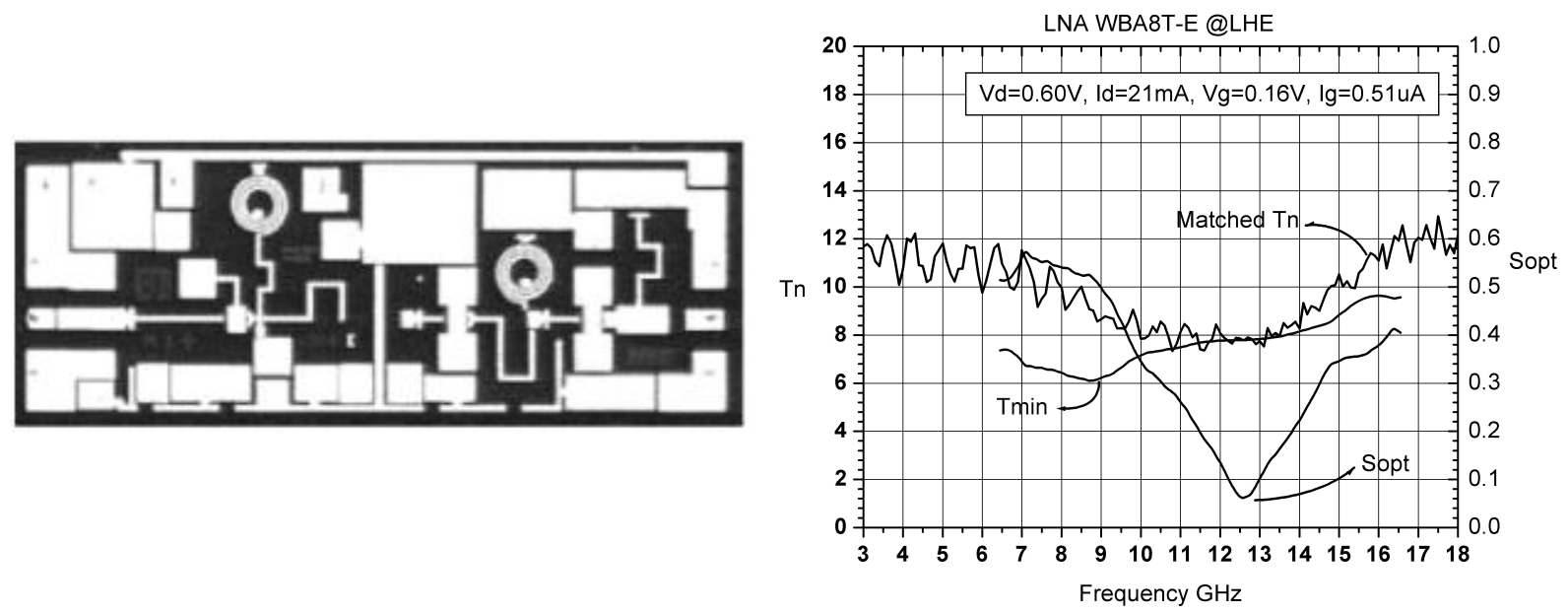

Figure 15: LNA chip and its cryogenic noise performance (measured). The WBA8T-E MMIC, manufactured by TRW, contains $4 \mathrm{InP}$ HEMT stages. Using inductive feedback in the first stage to improve input match while minimizing noise, its gain exceeds $20 \mathrm{~dB}$ across the 6-18 GHz IF band, with a minimum noise temperature $\left(\mathrm{T}_{\min }\right)$ of less than $10 \mathrm{~K}$ when the amplifier is cooled to $4 \mathrm{~K}$. The plot shows the measured noise temperature with a $50 \mathrm{ohm}$ source ("Matched Tn") as well.

\section{ROOM TEMPERATURE IF WIDEBAND SPECTROMETER SYSTEM}

To be effective, the instrument must have an appropriate spectrometer backend to process the receiver's very wide bandwidth IF signal. Fortunately, for the redshift determination application, the expected CO emission lines should be quite broad (approximately $250 \mathrm{MHz}$ ), so high spectrometer resolution is not required. A viable solution to this problem is to use an array of medium-resolution spectrometers to cover the entire IF bandwidth. The 128 channel, $0.5-4.5 \mathrm{GHz}$ WASP II analog autocorrelation spectrometer developed by one of the authors (A. Harris) provides a nearly ideal combination of bandwidth and spectral resolution for the CO line search application ${ }^{2}$.

The IF system requires an array of IF downconverters which will divide up the IF band into segments and convert the frequency ranges of the segments to a range which matches the bandwidth of the WASP II autocorrelators. A system is being developed which uses three IF downconverter modules; the downconverters are heterodyne mixers which convert their lower sideband inputs to a $0.5-4.5 \mathrm{GHz}$ output range. Each module is a wideband unit so that each channel band will be completely determined by its low-pass filter and local oscillator frequency. A single channel downconverter prototype unit has been built and tested.

Before the LNA output signal is fed to the downconverter system, it must be further amplified by a series of roomtemperature, wideband IF amplifiers. A 2-20 GHz amplifier module consisting of three commercial MMIC amplifiers has been developed and tested; it provides a quite flat, $30 \mathrm{~dB}$ gain across its bandwidth, with a measured $\mathrm{T}_{\mathrm{n}}$ of less than $450 \mathrm{~K}$ at room temperature and less than $150 \mathrm{~K}$ if the module is cooled to $77 \mathrm{~K}$. 


\section{ACKNOWLEDGEMENTS}

This work was supported in part by NASA/JPL and its Center for Space Microelectronics Technology, by NASA grants NAG5-9493, NAG5-4890, NAGW-107, and NAG2-1068, by the NASA/USRA SOFIA instrument development program, and by the Caltech Submillimeter Observatory (NSF grant AST-9980846).

\section{REFERENCES}

1. A. Blain, I. Smail, R. Ivison, J.-P. Kneib, and D. Frayer, "Submillimeter Galaxies," Physics Reports (in press, publication expected in 2002). Preprint available at http://arxiv.org as astro-ph/0202228.

2. A. Harris and J. Zmuidzinas, "A wideband lag correlator for heterodyne spectroscopy of broad astronomical and atmospheric spectral lines," Review of Scientific Instruments, 72\#2, 1531. 2001.

3. J. Kawamura, J. Chen, D. Miller, J. Kooi, J. Zmuidzinas, B. Bumble, H. LeDuc, and J. Stern, "Low-noise submillimeter-wave NbTiN superconducting tunnel junction mixers," Applied Physics Letters, 75, 4013. 1999.

4. S. Withington, G. Yassin, J. Leech, and K. Isaak, "An accurate expression for the input impedance of one-sided microstrip probes in waveguide," Proc. Tenth International Symposium on Space Terahertz Tech. 1999.

5. Y-C Leong and S. Weinreb, "Full-band waveguide-to-microstrip probe transitions," Microwave Theory and Techniques, Digest of Papers, IEEE. 1999.

6. J. Ward, F. Rice, and J. Zmuidzinas, "SuperMix: a flexible software library for high-frequency circuit simulation, including SIS mixers and superconducting components," Proc. Tenth International Symposium on Space Terahertz Tech. 1999.

7. F. Rice, J. Ward, J. Zmuidzinas, and G. Chattopadhyay, "Fast harmonic balance of SIS mixers with multiple junctions and superconducting circuits," Proc. Tenth International Symposium on Space Terahertz Tech. 1999. 